CERN-PH-TH/2004-011

NSF-KITP-04-09

hep-th/yymmnnn

\title{
Unconventional scenarios and perturbations therein
}

\author{
G. Veneziano ${ }^{1}$ \\ Department of Physics, CERN, Theory Division, CH-1211 Geneva 23, Switzerland
}

\begin{abstract}
After introducing a class of string-inspired unconventional cosmologies, I will discuss the spectra of adiabatic scalar and tensor perturbations therein. On the basis of a toy model I will argue that those spectra are quite likely too "blue" to be responsible for the observed large-scale structure of our Universe. I will then discuss a possible way out of this impasse whereby a scale-invariant spectrum of adiabatic density fluctuations emerges through the early decay of the Kalb-Ramond axion of string theory.
\end{abstract}

PACs : $11.25-\mathrm{w}, 98.80 . \mathrm{Bp}, 98.80 . \mathrm{Cq}$

\footnotetext{
${ }^{1}$ e-mail address: Gabriele.Veneziano@cern.ch
} 


\section{String-inspired unconventional cosmologies}

By present standards any reasonable cosmological model must include an inflationary phase in order to avoid the well-known problems of the old hot big bang (BB) scenario. Indeed, a sufficiently long phase of accelerated expansion easily takes care of the bad consequences of a generic (i.e. non-fine-tuned) initial state. Thus, for this talk, I will refer to Conventional Cosmologies as those evolving from a BB singularity (or from a Planckian phase) towards a regime of low curvature, density, and temperature through a long, slowroll inflationary phase. Besides solving the above-mentioned problems, slow-roll inflation also provides a quasi scale-invariant spectrum of primordial adiabatic density perturbations that appears to be compatible with cosmic microwave background (CMB) and large-scalestructure (LSS) data. Unfortunately, slow-roll inflation does not appear to be an easy outcome of our present, leading candidate for a theory of quantum gravity, superstring theory [1]; certainly it is not one of its generic properties (see, however, [2] for some interesting recent attempts at getting quasi-de-Sitter cosmology in string theory, and [3] for some ideas on how to even make it all sound very natural). By contrast, I will refer to (string-inspired) Unconventional Cosmologies as to those scenarios in which a non-singular version of the $\mathrm{BB}$ is preceded by a long phase of growing spacetime curvature $R$. Thus, while conventional cosmologies exhibit a monotonically decreasing $R$, unconventional ones, by definition, are characterized by a bounce in $R$.

Whether or not the curvature-bounce corresponds to a bounce in the scale-factor $a$, i.e. to contraction followed by expansion, depends both on the particular cosmology considered and on the conformal frame used. If the so-called Einstein frame (where distances are measured in Planck lengths, $\left.l_{\mathrm{P}}\right)$ is used, the pre-bang phase is always a contracting phase. However, if distances are measured in string-length $\left(l_{s}\right)$ units (the so-called string frame) the E-frame contraction can become an inflationary expansion, since $l_{\mathrm{P}} / l_{s}$ can be growing fast in that era. This is indeed one feature by which the old pre-big bang (PBB) scenario [4] differs from the ekpyrotic/cyclic (EKP) alternative [5]. In the latter, the ratio $l_{\mathrm{P}} / l_{s}$ actually decreases in time before the bounce, and thus the Universe contracts even in the string frame. Furthermore, the dilaton is reinterpreted, at all times, as the size of an extra dimension of space (as in the Horava-Witten picture [6]), a suitable dilaton potential is added, and the bounce occurs at vanishing (or at very small) coupling. In the PBB framework there are a priori many possibilities, but the standard scenario is one in which the pre-bang evolution proceeds from weak to strong coupling, with the bounce supposedly occurring at large curvature and coupling. Thus the extra dimension of space is only considered to be relevant, if at all, for the description of the bounce itself (see e.g. [7]).

Independently of the frame, in these unconventional cosmologies (as in standard inflation) even the largest scales we observe today have been in causal contact at least once in the past provided the pre-bang phase is sufficiently long. As stressed by many people (see e.g. [8]) what is needed to solve the cosmological problems is "generalized inflation", in the 
sense that $\dot{a}$ and $\ddot{a}$ have the same sign. Accelerated expansion and accelerated contraction are, a priori, equally viable. However, unlike in standard inflation, in a bouncing-curvature cosmology, the shorter the scale the shorter the distances it probes at its exit from the horizon $H^{-1} \sim R^{-1 / 2}$. Put differently, while in standard inflation Planck (or string)-scale physics is only felt by scales that have been stretched out of our present horizon -and are thus unobservable - in cosmologies with a curvature bounce very short distance physics is probed by scales that are much inside the horizon -and thus observable- today (e.g. gravity waves at wavelengths accessible to operating antennas or interferometers).

The good news is that cosmologies of this unconventional type are easy to get in string theory; they are even natural, thanks to the ( $T$-duality-like) symmetries of the stringcosmology equations [9]. Of course, this is only true modulo finding a solution to the exit problem, i.e. modulo the assumption that, indeed, the big bang singularity can be avoided (or reinterpreted) so that continuation from $t>0$ to $t<0$ becomes possible. This latter problem is not an easy one to tackle since it demands control on string theory in the time-dependent, strong-curvature regime, for which adequate techniques are still lacking. Unfortunately, the problem is not just a conceptual one: as we have mentioned, the strongcurvature phase (the bounce itself) is in principle accessible to observation, meaning that its details affect physical observables. This is certainly the case for short-scale perturbations; however, it is not completely obvious that large-scale observables are completely insensitive to the details of the bounce. These details are most likely to affect the way various perturbation variables (mis)match across the bounce itself. It has been argued [10] that imposing different matching conditions at the bounce results in completely different perturbation spectra even at the largest scales. And this is precisely where the relative merits of conventional and unconventional cosmologies will be pinned down, eventually.

In the absence of general results on this issue I will discuss (Section 2) a model that implements, in an elegant (and possibly even physical) way, a non-singular curvature bounce. I will compute tensor and scalar perturbations therein, discuss the tilted spectra it leads to, and the matching conditions it suggests to be valid. Within such a setup there is no way to reproduce the CMBR (and LSS) data other than invoking the so-called curvaton mechanism, a way to convert a flat spectrum of isocurvature (entropy) perturbations (that naturally reside in the Kalb-Ramond axion field of string theory) into the desired curvature perturbation one needs phenomenologically. This will be the contents of Section 3.

\section{Conventional perturbations in unconventional cosmologies}

The question we would like to address is simple: What is the spectrum of (standard, adiabatic) scalar (S) and tensor (T) perturbations in a bouncing-curvature cosmology of the $\mathrm{PBB}$ or EKP type? The literature on the subject contains different claims:

- Proponents of the PBB scenario argued long ago [11], [12] that both $\mathrm{S}$ and T- 
perturbations are produced with strongly tilted, blue spectra $\left(n_{S}>1, n_{T}>0\right.$ in standard notation). If so, these perturbations are irrelevant to CMB anisotropy or LSS.

- Proponents of the EKP scenario have argued instead in favour of a blue, tilted spectrum of $\mathrm{T}$ perturbations $\left(n_{T}>0\right)$ and of a quasi-scale-invariant spectrum of $\mathrm{S}$ perturbations $\left(n_{S} \sim 1\right)[13]$.

The reasons for this discrepancy can be traced to the fact that tensor perturbations (gravity waves) are conceptually simpler to analyse because they are automatically gaugeinvariant. Even then, some assumption is needed on how the GW amplitude behaves across the bounce. As it turns out, this only produces a possible "logarithmic disagreement" in the final T spectra (cf. Refs. [11] and [14]). By contrast, naive scalar metric perturbations are affected by gauge-invariance problems and, as a result, different gauge-invariant $\mathrm{S}$ perturbations can be defined. In the case we shall discuss here, that of a single scalar field, all of them are related, and thus contain the same physical information. Nonetheless, different assumptions as to which of them behave smoothly through the bounce, and which do not, lead to different physical predictions on present observations. The following gauge-invariant variables are particularly popular:

- The Bardeen potential $\Psi$, corresponding to curvature perturbation on shear-free hypersurfaces. It is much in use because it is also the metric perturbation in the longitudinal (or Newtonian) gauge, in which equations are particularly simple [15]. It is also directly related to $\Delta T / T$ via the Sachs-Wolfe effect.

- The curvature perturbation on comoving (here constant $\varphi$ ) hypersurfaces, usually denoted by $\mathcal{R}$.

- The curvature perturbation on constant-density hypersurfaces, usually denoted by $\zeta$.

On super-horizon scales, $\mathcal{R}$ and $\zeta$ are very close to one another and are constant in time in the absence of entropy perturbations (more technically, in the absence of non-adiabatic pressure perturbations). This is why they are also much used in standard slow-roll inflation, particularly from the end of inflation till decoupling. However, in standard inflation, $\Psi$ and $\mathcal{R}$ behave very similarly during the inflationary phase: both of them are described well by a constant mode that dominates over a rapidly decaying mode.

In the bouncing cosmologies that we are discussing the situation is much less clear since the decaying mode may become a growing mode for certain kinds of bounces and/or for certain perturbation variables. In the models at hand, for instance, $\mathcal{R}$ and $\zeta$ have tilted (blue) spectra, just like the tensor modes, while $\Psi$ can have a red or flat spectrum, depending on the scenario. In the PBB scenario, $\Psi$ is red $(n=0)$ and its modes grow so large outside the horizon that linear perturbation theory breaks down in the longitudinal/Newtonian 
gauge even before reaching Planckian curvatures. However, this was shown in [12] to be just a problem of the longitudinal gauge, possibly related to an insufficient damping of shear in these cosmologies. In other gauges perturbation theory remains valid since the metric entries contain extra powers of $k \eta \ll 1$. In the ekpyrotic scenario the addition of a suitable potential turns the red spectrum of $\Psi$ into an almost flat one $(n \sim 1)$ like that of slowroll inflation. The spectrum of $\mathcal{R}$, however, remains blue. As a result: if $\mathcal{R}$ is continued smoothly past the bounce and then is used to compute $\Psi$ at $t>0$, the flat mode of $\Psi$ is found to decay away at late time, leaving behind a blue spectrum; if instead some other variable is assumed to behave smoothly then (part of) the scale-invariant mode of $\Psi$ can be transmitted through the bounce. These ambiguities have led to a long series of articles making contradictory claims [16].

In order to clarify this issue we have looked into a model [17] with just metric and dilaton and with a regular bounce in $R$. The bounce is obtained via a (general-covariant) non-local potential. Homogeneous solutions and their perturbations can be followed through the bounce both analytically and numerically. Before describing the model in some detail the following two warnings ought to be added:

- For the moment we considered just a class of models. General lessons should not be drawn too fast;

- More complicated cases involving non-adiabatic pressure perturbations (by adding for instance a perfect-fluid component) are expected to behave differently and are under investigation.

Our non-singular bouncing-curvature Universe is a solution of the following covariant, non-local action (written here in the string frame):

$$
S=-\frac{1}{2 l_{s}^{d-1}} \int d^{d+1} x \sqrt{|g|} e^{-\varphi}\left[R+(\nabla \varphi)^{2}+V(\bar{\varphi})\right]
$$

(metric conventions: $+---\ldots$ ). Here the potential $V(\bar{\varphi}(x)$ ), a local function of $\bar{\varphi}$, is instead a non-local function (yet a scalar under general coordinate transformations) of the dilaton according to the definitions:

$$
V=V\left(e^{-\bar{\varphi}}\right), \quad e^{-\bar{\varphi}(x)}=\int \frac{d^{d+1} y}{\lambda_{\mathrm{s}}^{d}} \sqrt{|g(y)|} e^{-\varphi(y)} \sqrt{\partial_{\mu} \varphi(y) \partial^{\mu} \varphi(y)} \delta(\varphi(x)-\varphi(y)) .
$$

An example of background (representative of a large class of non-singular cosmologies), based on $V(\bar{\varphi})=-V_{0} e^{4 \bar{\varphi}}$, is

$$
a(\tau)=\left[\tau+\sqrt{\tau^{2}+1}\right]^{1 / \sqrt{3}}, \quad \bar{\varphi}=-\frac{1}{2} \ln \left(1+\tau^{2}\right)+\varphi_{0}, \quad \tau=t / t_{0},
$$

This background corresponds to a cosmology in which both $R$ and $\bar{\varphi}$ evolve in time following a bell-shaped curve. It is characterized by two parameters: the coupling and curvaturescale at $t=0$ : the latter also gives the duration of the bounce. The nice thing is that the 


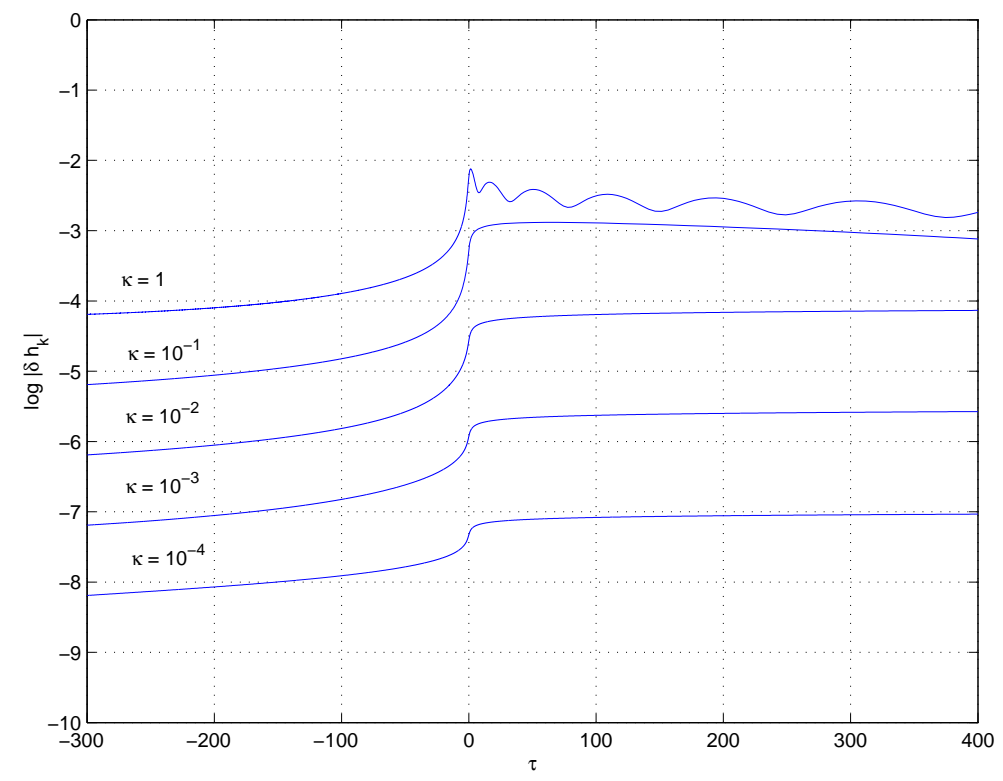

Figure 1: Tensor perturbations evolving across the bounce for different values of the comoving wave-number $k$ (in units such that $t_{0}=1$ ).

perturbations equations can be solved numerically and thus fluctuations can be followed from beginning to end. The results are shown in Figs. 1-5, which I will now comment upon.

Figures 1 and 2 concern tensor perturbations. In the former we can see the evolution of perturbations across the bounce for different scales, and their amplification while they are outside the horizon. In the latter the amplification is made quantitative by studying, for each $k$, the Bogolubov coefficients $\alpha$ and $\beta$. As a check of the numerical precision, the probability conservation equation $|\alpha|^{2}+|\beta|^{2}=1$ is also tested. Fig. 3 is the analogue of Fig. 1 for the scalar perturbation $\delta_{\mathcal{R}} \equiv k^{3 / 2} \mathcal{R}$, while, for lack of space, we do not present the analogue of Fig. 2. In Fig. 4 the Bogolubov coefficient $\beta$ is fitted (both for $\mathrm{T}$ and $\mathrm{S}$ perturbations) to theoretical curves predicting parabolas in a plot against $\log k$. The (three-parameter) fits are clearly excellent. Finally, in Fig. 5 we compare the behaviours of $\Psi$ and $\mathcal{R}$ across the bounce. This shows that only the latter variable behaves smoothly around $t=0$. If a little region around the bounce, $-\epsilon<t<\epsilon$, is removed, and continuity is assumed between the values of various perturbations (and their time derivatives) at $t= \pm \epsilon$, a much larger error is made for $\Psi$ than for $\mathcal{R}$.

The conclusion appears to be that, at least in this class of models, continuity across the bounce should be imposed on the perturbation variable $\mathcal{R}$ and not on the Bardeen potential $\Psi$. As is well known, in such a case the final spectrum of adiabatic scalar perturbation follows that of tensor perturbations and, unfortunately, they are both strongly tilted in favour of 


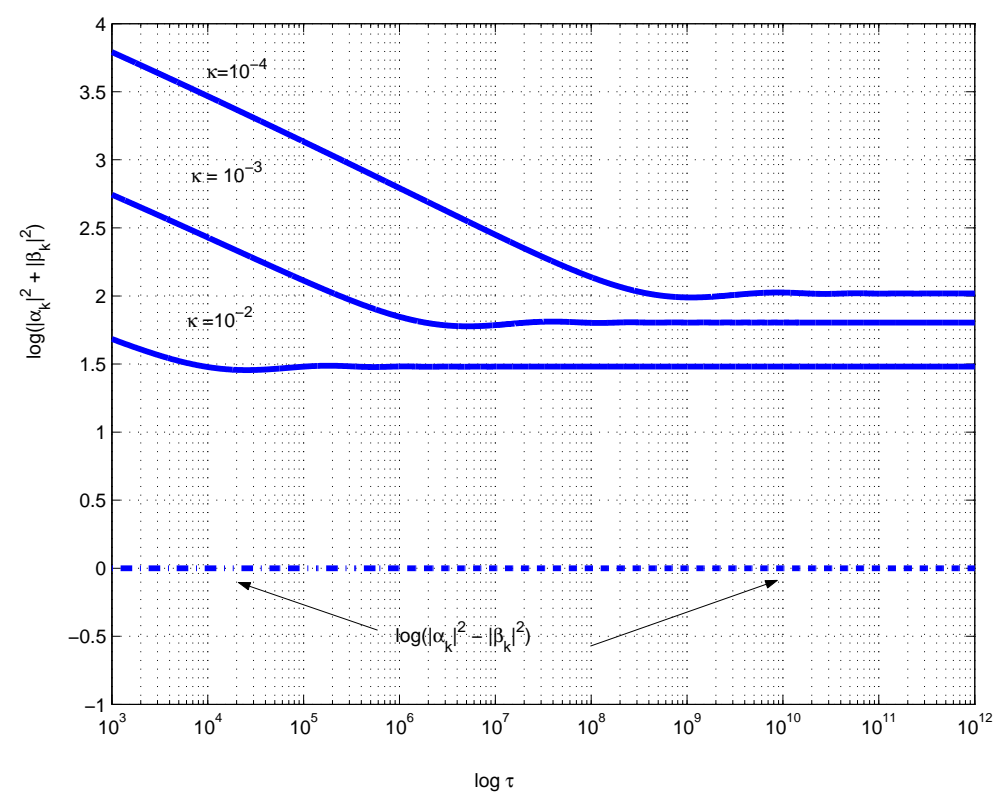

Figure 2: Numerical evaluation of the Bogolubov coefficients for tensor perturbations.



Figure 3: Evolution of $\delta_{R}$ for different values of the comoving wave-number. 


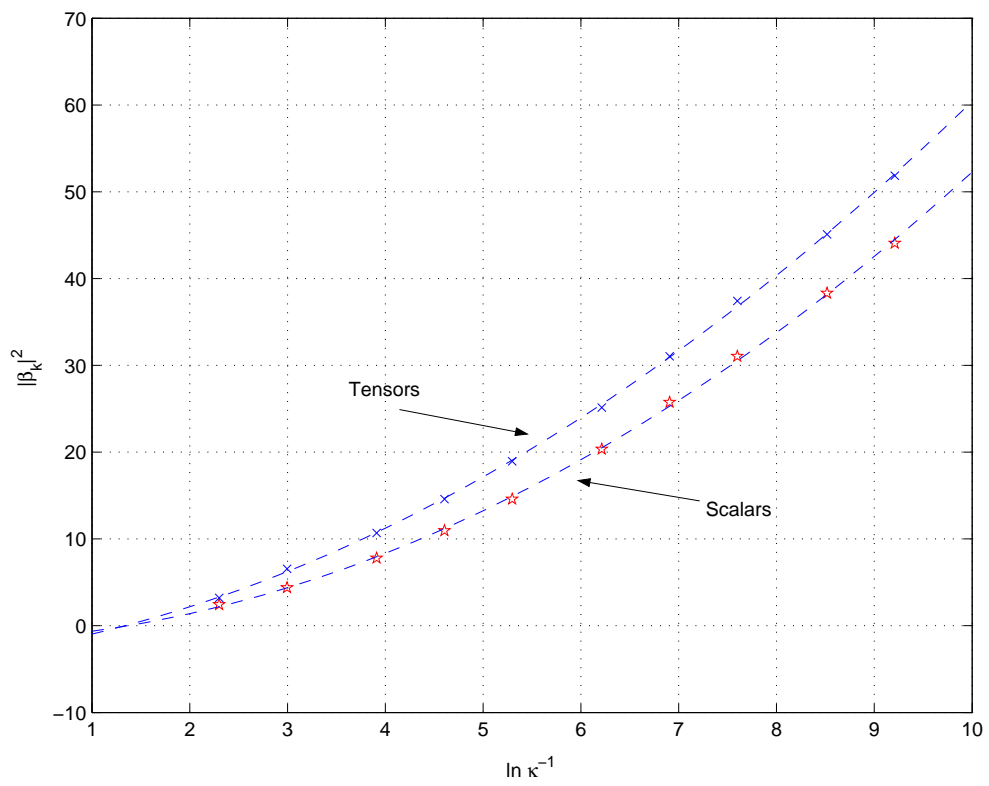

Figure 4: Fit of S and T Bololubov coefficients to theoretical expectations.

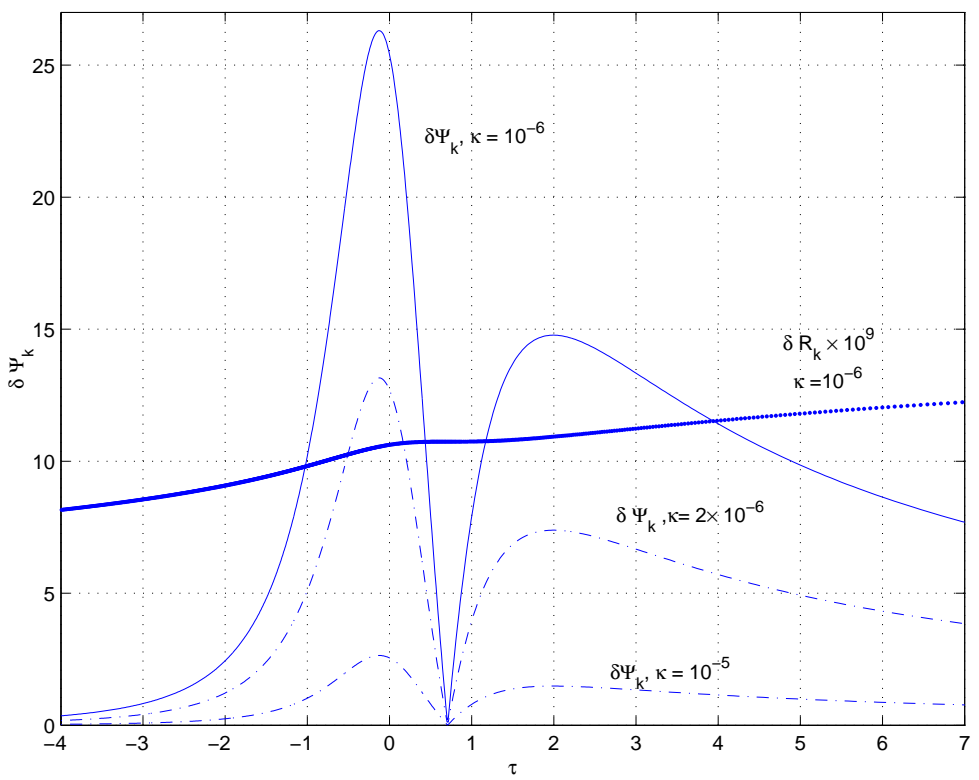

Figure 5: Evolution of $\Psi$ and $\mathcal{R}$ across the bounce. 
short wavelengths.

In the rest of this talk I will assume this to be the general case for cosmologies with a curvature bounce and will ask: Would this doom forever PBB- or EKP-type cosmologies by making them unable to generate an interesting spectrum of large-scale fluctuations? In the next section I will argue that this is not necessarily the case, thanks to an unconventional mechanism to convert isocurvature perturbations into adiabatic density (and curvature) perturbations, which has become known as the "curvaton" mechanism.

\section{Unconventional perturbations in string cosmology}

Let me start by recalling the spectra of various kinds of massless perturbations in string cosmology, limiting myself to the standard PBB scenario in which the pre-bounce phase contains just non-trivial metric and dilaton backgrounds and there is no dilaton potential. In this case, during the pre-bang phase only the dilaton perturbations couple to (scalar) metric perturbations: in standard terminology they represent adiabatic (curvature) perturbations while those of all other fields are isocurvature (entropy) perturbations. For a complete analysis we refer to [18]. We just recall here that

- Under the assumptions made in the previous section, tensor as well as scalar-dilaton perturbations have tilted spectra with $n_{T}=3$ and $n_{S}=4$ respectively. Such a prediction is very robust: for instance, does not depend on the evolution of the six extra dimensions during the pre-bounce era.

- Electromagnetic (and more generally gauge-field) perturbations are not as blue but they are still non-scale-invariant. Their spectral index depends on the evolution of extra dimensions and on the details of the embedding of $U(1)_{\mathrm{em}}$. The whole effect is due to the time-variation of the fine-structure constant $\alpha$ and is thus characteristic of theories in which $\alpha$ is replaced by a dynamical field. An interesting, and yet unsolved, issue is whether such fluctuations can act as seeds for the observed cosmic magnetic fields.

- The Kalb-Ramond axion of string theory (i.e. the SUSY partner of the dilaton) can have a blue, red or flat spectrum depending on the evolution of the six extra dimensions [19].

More explicitly, the axion-fluctuation spectrum reads

$$
\left|\delta \sigma_{k}\right|^{2} \equiv k^{3}\left|\sigma_{k}\right|^{2}=\left(\frac{H^{*}}{M_{\mathrm{P}}}\right)^{2}\left(\frac{\omega}{\omega^{*}}\right)^{n-1}, \quad(4-2 \sqrt{3}) \sim 0.53<n<2,
$$

where $H^{*}$ is the maximal value reached by the Hubble parameter just before the bounce, and is thus expected to be of the order of the string scale $M_{s}=l_{s}^{-1}, \omega^{*}$ is that same scale 
redshifted to the present time (and thus of order $10^{11} \mathrm{~Hz}$ ) and $\sigma M_{\mathrm{P}}$ is the canonically normalized axion field; $n$, the axion's spectral index, has a large possible range of values centred around the scale-invariance point $n=1$. Scale invariance is reached for a particularly symmetric evolution [18] of all nine spatial dimensions (modulo $T$ duality transformations of the internal space). There could be theoretical reasons for picking up that point since some of the cosmologies appear to be exact, flat-spacetime solutions from the 11-dimensional viewpoint.

Unfortunately, even if we assume that string theory selects the $n=1$ solution, the resulting perturbations fail to reproduce the structure of acoustic peaks now clearly observed by WMAP and other experiments [20]. The reason for this is that axion perturbations, being isocurvature, feed back on metric (curvature) perturbations to 2nd order but give a large displacement in the position of the acoustic peaks [21].

At this point the "curvaton" mechanism [22] comes to the rescue. It says that, if a potential is generated for $\sigma$ (as it should, as a result of Peccei-Quinn-symmetry breaking), and if $\sigma$ does not come out of the bounce sitting at its minimum (and there is no reason why it should), then the axion perturbation can be converted into adiabatic curvature perturbations when the axion decays. For this to work, we need the axion to decay (and it should do so before primordial nucleosynthesis so as not to spoil its successes) after having become a non-negligible component in the total energy.

The conversion efficiency can be computed (see e.g. [23]). If axion dominates completely at decay, the Bardeen potential generated after the decay is:

$$
k^{3}\left|\Psi_{k}\right|^{2}=f^{2}\left(\sigma_{i}\right)\left|\delta \sigma_{k}\right|^{2}=f^{2}\left(\sigma_{i}\right)\left(\frac{H^{*}}{M_{\mathrm{P}}}\right)^{2}\left(\frac{\omega}{\omega^{*}}\right)^{n-1}, \quad f\left(\sigma_{i}\right) \sim\left(0.13 \sigma_{i}+0.18 / \sigma_{i}\right)>0.3
$$

where $\sigma_{i}$ is the initial displacement of the axion from the minimum of the potential and the result is diluted, in general, by the fraction $\Omega_{\sigma}$ of the total energy density carried by the axion at decay. The spectrum given in (3.3) can be converted into a prediction for the celebrated CMB multipoles $C_{l}$. In particular, for $n, \sigma_{i} \sim 1$ :

$$
C_{2}=\alpha_{n}^{2} f^{2}\left(\sigma_{i}\right)\left(\frac{H^{*}}{M_{\mathrm{P}}}\right)^{2}\left(\frac{\omega}{\omega^{*}}\right)^{n-1}, \alpha_{n}^{2} \sim \frac{1}{54 \pi}, f\left(\sigma_{i}\right)^{2} \sim 0.1
$$

to be compared with COBE's normalization [24]: $C_{2}=(1.09 \pm 0.23) \times 10^{-10}$.

As it turns out, fitting COBE's normalization is the most severe constraint. Once this is fulfilled, the acoustic-peak structure comes out fine, provided the primordial axion spectrum is nearly flat $(n \sim 1)$. The PBB parameter space consistent with CMB observations is sketched in Figs. 6 and 7. In Fig. 6 a possible break $\delta$ in the tilt has been inserted above the acoustic-peak scale and the straight lines correspond to the indicated values for $\log \left(H^{*} / M_{\mathrm{P}}\right)$. The vertical band is the allowed region. In Fig. 7, the allowed region (in 


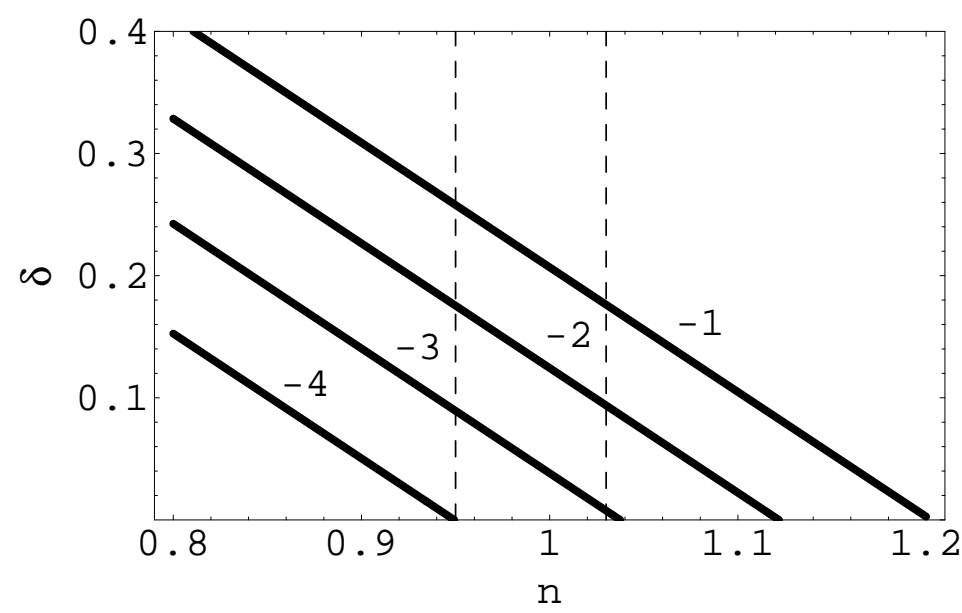

Figure 6: Allowed region in $\delta$ and $\left(H^{*} / M_{P}\right)$

$n)$ is shown as a function of $\left(H^{*} / M_{\mathrm{P}}\right)$ and $\sigma_{i}$. A particularly simple solution, given by $n=1, \delta=0, \sigma_{i}=1, H^{*} / M_{\mathrm{P}} \sim 5 \times 10^{-4}$, illustrates here a general fact: slightly blue spectra and/or low ratio $\left(H^{*} / M_{\mathrm{P}}\right)$ (i.e. a low $M_{s} / M_{\mathrm{P}}$ if $H^{*} \sim M_{s}$ ) are preferred. At first sight it would look as if an extra parameter to play with for normalization purposes would be $\Omega_{\sigma}$. It turns out, however [25], that making $\Omega_{\sigma}$ too small increases the non-gaussianity of the perturbation beyond present experimental bounds. A measurable amount of nongaussianity would favour, on the other hand, curvaton-like models with respect to more conventional scenarios.

Summarizing:

- A non-singular bouncing curvature model supports the old PBB claim of blue S and $\mathrm{T}$ perturbation spectra rather than the EKP claim of flat $\mathrm{S}$ and blue $\mathrm{T}$ perturbations.

- It also supports previous claims about the decoupling of large-scale phenomena from the details of the high-curvature bounce.

- The best way to rescue the phenomenological viability of bouncing cosmologies appears to be adding a certain amount of non-adiabaticity, like in the curvaton scenario. Possibly, a non-vanishing $\delta p_{n a}$ is implicit in the EKP prescription on how to go over the bounce.

- String theory has a good curvaton candidate, the Kalb-Ramond axion. The parameter space is constrained by existing data (particularly after WMAP).

- Detailed predictions are different from those of standard slow-roll inflation (small tensor component, possibly large non-gaussianity and/or isocurvature component) ... and can/will be tested. 


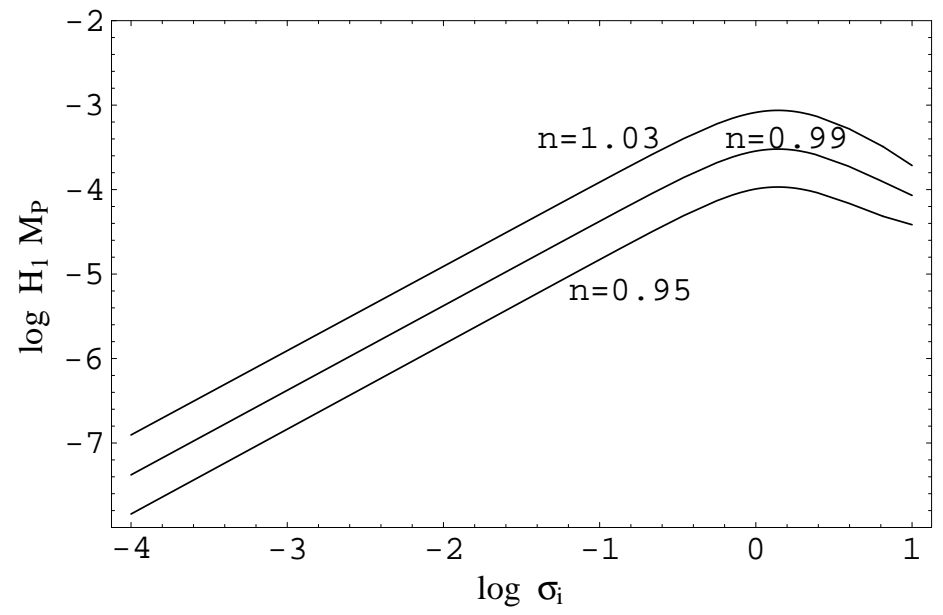

Figure 7: Allowed region in $\sigma_{i}$ and $\left(H^{*} / M_{\mathrm{P}}\right)$

This research was supported in part by the National Science Foundation under Grant No. PHY99-07949.

\section{References}

[1] Brustein, R. and Steinhardt, P. J., Phys. Lett. B302, 196 (1993).

[2] Kachru, S., Kallosh, R., Linde, A. and Trivedi, S. P., Phys. Rev. D68, 046005 (2003);

Kachru, S. et al., JCAP 0310, 013 (2003).

[3] Susskind, L., this conference.

[4] Gasperini, M. and Veneziano, G., Phys. Rep. 373, 1 (2003);

see also Lidsey, J. E., Wands, D. and Copeland, E. J., Phys. Rep. 337, 343 (2000).

[5] Khouri, J., Ovrut, B. A., Steinhardt, P. J. and Turok, N., Phys. Rev. D66, 046005 (2002); Khouri, J., Ovrut, B. A., Seiberg, N., Steinhardt, P. J. and Turok, N., Phys. Rev. D65 (2002) 086007; Steinhardt, P. J. and Turok, N., Phys. Rev. D65 (2002) 126003.

[6] Horava, P. and Witten, E., Nucl. Phys. B460, 506 (1996); ibid. B475, 94 (1996).

[7] Maggiore, M. and Riotto, A., Nucl. Phys. B548, 427 (1999).

[8] Turner, M., this conference.

[9] Veneziano, G., Phys. Lett. B265, 287 (1991).

[10] Durrer, R. and Vernizzi, F., Phys. Rev. D66, 083503 (2002). 
[11] Brustein, R., Gasperini, M., Giovannini, M. and Veneziano, G., Phys. Lett. B361, 45 (1995).

[12] Brustein, R., Gasperini, M., Giovannini, M., Mukhanov, V. and Veneziano, G., Phys. Rev. D51, 6744 (1995).

[13] Khouri, J., Ovrut, B. A., Steinhardt, P. J. and Turok, N., Phys. Rev. D66, 046005 (2002); Gratton, S., Khouri, J., Steinhardt, P. J. and Turok, N., astro-ph/0301395; Tolley, A. J., Turok, N. and Steinhardt, P. J., hep-th/0306109.

[14] Boyle, L. A., Steinhardt, P. J. and Turok, N., hep-th/0307170.

[15] Mukhanov, V. F., Feldman, H.A. and Brandenberger, R. H., Phys. Rep. 215, 203 (1992).

[16] Lyth, D. H., Phys. Lett. B526, 173 (2002); ibid. B524, 1 (2002); Brandenberger, R. and Finelli, F., JHEP 11, 056 (2001); Martin, J., Peter, P., Pinto-Neto, N. and Schwarz, D. J., Phys. Rev. D65, 123513 (2002); Finelli, F., JCAP 0310, 011 (2003).

[17] Gasperini, M., Giovannini, M. and Veneziano, G., Phys. Lett. B569, 113 (2003); hepth/0401112.

[18] Buonanno, A., Meissner, K. A., Ungarelli, C. and Veneziano, G., JHEP 9801, 004 (1998); Brustein, R. and Hadad, M., Phys. Rev. D57, 725 (1998).

[19] Copeland, E. J., Easther, R. and Wands, D., Phys. Rev. D56, 874 (1997); Copeland, E. J., Lidsey, J. E. and Wands, D., Nucl. Phys. B506, 407 (1997).

[20] Spergel, D. et al., Astrophys. J. Suppl. 148, 175 (2003); Lee, A. T. et al., Astrophys. J. 561, L1 (2001); de Bernardis, P. et al., Astrophys. J. 564, 559 (2002); Halverson, N. W. et al., Astrophys. J. 568, 38 (2002); Netterfield, C. B. et al., Astrophys. J. 571, 604 (2002); Benot̂t, A. et al., Astron. Astrophys. 399, L19-L23 (2003).

[21] Durrer, R., Gasperini, M., Sakellariadou, M. and Veneziano, G., Phys. Lett. B436, 66 (1998); Phys. Rev. D59, 43511 (1999); Melchiorri, A., Vernizzi, F., Durrer, R. and Veneziano, G., Phys. Rev. Lett. 83, 4464 (1999).

[22] Mollerach, S., Phys. Rev. D42, 313 (1990); Moroi, T. and Takahashi, T., Phys. Lett. B522, 215 (2001); Enqvist, K. and Sloth, M. S., Nucl. Phys. B626, 395 (2002); Lyth, D. H. and Wands, D., Phys. Lett. B524, 5 (2002).

[23] Bozza, V., Gasperini, M., Giovannini, M. and Veneziano, G., Phys. Lett. B543, 14 (2002); Phys. Rev. D67, 063514 (2003); Sloth, M. S., Nucl. Phys. B656, 239 (2003).

[24] Smoot, G. F. et al., Astrophys. J. 396, L1 (1992); Bennett, C. L. et al., Astrophys. J. Lett. 464, L1 (1996); Tegmark, M., Astrophys. J. Lett. 464, L35 (1996).

[25] Lyth, D., Ungarelli, C. and Wands, D., astro-ph/0208055. 